\title{
Fuzzy Modeling for Wireless Sensor Networks
}

\author{
A.H. Mohamed \\ Solid State Dept., \\ National Centre for Radiation \\ Research \& Technology (NCRRT), \\ Atomic Energy Authority, Cairo, Egypt
}

\begin{abstract}
Applications of the wireless sensor networks have widely increased in recent years. However, a lot of work has been developed to improve the performance of these wireless sensor networks. But, there are some main limitations till now due to complexity appeared for maximizing the lifetime, dealing with the noisy data, having a balance of the node loading, speeding up the transmission process of wireless sensor networks (WSNs). The present work proposes a new routing technique that has used the fuzzy modeling to overcome these drawbacks of WSNs. Proposed fuzzy technique seeks to determine the optimal route path from source to destination so that the energy consumption is balanced and minimized. The proposed fuzzy routing technique is applied for a WSN used in radiated products' system. Then, the suggested system is compared with Traditional Low- Energy Adaptive Clustering Hierarchy (LEACH) commonly used protocol for WSNs. The obtained results show significant increase in the performance of the WSNs. However, the proposed system has proved its well suitability for the real-time applications.
\end{abstract}

\section{Keywords}

Fuzzy Modeling, Wireless Sensor Networks.

\section{INTRODUCTION}

In recent years, there are great needs for observing and monitoring the surrounding environments for many military and scientific applications.

This drives to design the wireless sensor networks those are special class of ad-hoc networks. A wireless sensor network consists of a high number of small, inexpensive, self-powered devices called sensor nodes [1]. Each sensor node can sense, compute, and communicate with other devices to collect information about a physical environment [2].

WSNs are differenced about the ad-hoc networks in some properties. WSNs have more severe energy constraints, much larger density of sensor nodes, and lower cost. Another important property of WSNs is its ability for gathering information rather than distributed computing of the ad-hoc networks [3].

The energy plays the most important role in WSNs' operation to have a reliable, efficient uses and long lifetime. Therefore, various routing and energy-saving protocols for WSNs have been proposed [4-6]. Clustering routing algorithms represent a great area of these protocols to perform energy-efficient routing and extend the lifetime of the WSNs. Its main principles based on the energy consumption can be decreased by using only a part of the nodes that has a cluster head. However, the communication between this group of nodes and the base station is executed by their cluster head. Where, the data sent by each node is then collected by cluster heads, compressed and transmitted to the base station via the cluster heads only [7].
It is found that, Low- Energy Adaptive Clustering Hierarchy $(\mathrm{LEACH})$ is a popular hierarchical clustering protocol used for WSNs. It is a localized clustering method based on a probability model. It decomposes the WSN into group of clusters. Each cluster has a head. The cluster heads are used as routers to the sink node [8]. LEACH uses a probabilistic method to determine its cluster-heads $(\mathrm{CHs})$. It applies the randomized rotation to have a balanced energy consumption of the cluster-heads over the network. LEACH uses singlehop routing. So, each node transmits data directly to its cluster head [9]. Although, LEACH has introduced considerable success, it still suffers from some problems such as:

1- it uses fixed (crisp) values for determining the energyaware routing paths. This causes some difficulties in adapting the changes appeared in the sensor types [8]. Besides, complexity appeared at needs to have balanced values between conflict factors of the networks.

2- it is unsuitable to be applied for complex networks.

3- Sometimes, the random selection of the head clusters causes some problems such as: (1) there are no selected heads. (2) many heads can be selected in a single round. (3) the heads can be selected at a near distance. This can cause inefficient energy distribution [9].

To overcome these problems, the present research introduces a new solution that uses the fuzzy modeling for LEACH routing protocol in wireless sensor networks to maximize their lifetime and improve their performance.

The remaining of this paper is organized as follows: Section 2 deals with the fuzzy modeling. Section 3 presents the related work. While, a description of the proposed system is explained in section 4. Simulation results are presented in section 5. Section 6 concludes the work.

\section{FUZZY MODELING}

Modern technologies have applied the fuzzy logic successfully in many areas [10]. It provides a particularly good and less complex solution than the computational calculations for many engineering problems including communications and networks [11]. Fuzzy Logic can handle the uncertain or nosily inputs.

The operation of the fuzzy logic is based on the fuzzy sets, each of which may represent a linguistic term like "Cold", "Long", "Small", etc.

Fuzzy sets are represented by the range of real values over which the set is mapped, called domain, and the membership function.

A membership function assigns a truth value between 0 and 1 to each point in the fuzzy set's domain. Various types of fuzzy sets can be achieved based on the shape of the membership function, [12]. 
The operation of the fuzzy system consists of three main modules: Fuzzification, inference engine, and defuzzification modules as shown in fig. (1).

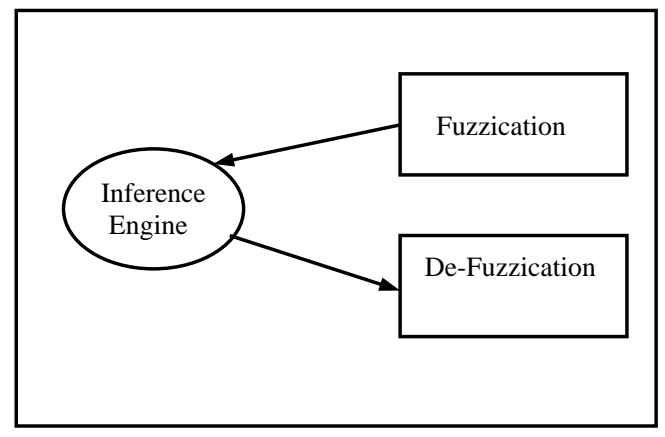

Fig. (1): Main Structure of the Fuzzy System

The Fuzzification module maps each crisp input value to degree of membership for each fuzzy set [13]. The Fuzzificated values are processed by the inference engine using its predefined rules. While, the linguistic variables output fuzzy variables are mapped into the crisp values by the defuzzifier. Common defuzzification techniques are centroid, composite maximum, composite mass, etc [14].

\section{RELATED WORK}

Researchers have developed different routing protocols for WSNs. In the same way, recently, several fuzzy-clustering routing protocols algorithms for WSNs have been developed. These clustering algorithms use the fuzzy logic to handle uncertainties and integrating different clustering parameters to have the cluster-heads.

Manjunatha et. al., proposed a fuzzy approach for clustering algorithm that can select the cluster heads at the base station [15]. While, Chang, et. al, suggested the uses of the minimum energy path [16]. Gu, S. et al. introduced the uses of maximum residual energy path with minimum number of hops to the sink [17].

Although these protocols have considerable success, they suffer from some drawbacks. For examples: using high energy by the nodes for processing and sending the information. Therefore, more work is required to overcome the limitations of the WSNs.

\section{THE PROPOSED SYSTEM}

Recently, wireless sensor networks have represented a great area of real time applications. Researchers have introduced a lot of work to deal with the needs for this great wide spreading of WSNs. But, there are some open problems till now. Some of these problems are: balancing between the conflict factors of the networks, minimizing the energy losses by selecting the most suitable routing path, increasing the WSNs' lifetime and dealing with uncertain data.

The present work suggests incorporating the fuzzy logic modeling in the cluster protocol algorithms as a novel solution for the previous problems. Suggested system uses the fuzzy modeling for LEACH protocol that is a commonly used cluster protocol algorithm as a case of these cluster protocols those suffered from all of the previous problems.

The proposed system is a localized clustering system based on the fuzzy modeling. Figure (2) represents the main structure of the WSN used by the proposed system where the link between the base node and the cluster heads is described.

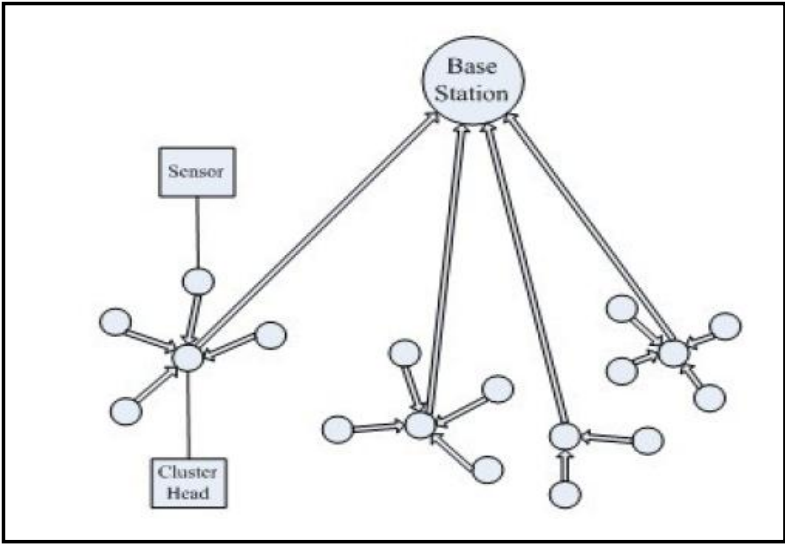

Fig. (2) : The link between the Base Node and the Head Clusters Only in the proposed system

The operation of the proposed system is executed according to three steps of the fuzzy system that shown in fig. (1). The core module of the proposed fuzzy system is the inference module. To execute its tasks, it has applied the Mamdani's method for the inference process [18]. This method is usually used in many applications because of its simple structure of 'min-max' operations. The proposed system has executed main tasks, as:

Firstly, fuzzy modeling has proved its goodness and well suits for applications those having conflict requirements as networks. Whereas, designing and operating the WSNs involve many conflict factors. For example, lower transmission power vs. longer transmission duration, multihop vs. direct communication, computation vs. communication etc.

Therefore, the suggested system introduces uses the fuzzy modeling to integrate these conflict parameters and determine an acceptable output from them. The fuzzy modeling can handle the conflicting situations and imprecision in data without needing for applying the complex mathematical modeling.

Secondly, Moreover, using the fuzzy modeling can easily adapt the changes of the energy values required for using different types or locations of the sensor nodes.

Thirdly, the proposed fuzzy logic system is used to select the optimum cluster heads required for having the lowest energy routing path of the WSN.

The fuzzy inference engine of the suggested system computes the node's fitness using the evaluated parameters to determine its suitability for uses as a cluster head node.

These evaluated parameters applied by the proposed system are: the energy level, concentration, and centrality parameters. Energy level is determined at each node in the network. The concentration is the number of neighbor nodes. While, the centrality is used to measure how close the node to the cluster head.

Based on the fitness values, the nodes are re-ordering in a list. Every round, the list is updated with the new selected heads. Also, the routing load balance for the sensor nodes is achieved based on the fuzzy rules. This drives to have the required energy for a maximum number of nodes to complete their operations. However, the lifetime of the WSNs is increased. 
For example, the proposed system is suggested two inputs for selecting the cluster-heads. They are: distance to the base station and residual energy of the cluster-head.

Figure (3) represents the linguistic variables for the distance to the base station input variable fuzzy set are close, medium and far. While, it uses a trapezoidal membership function for close and far. The membership function of medium is a triangular membership function. On the other side, figure (4) describes the fuzzy set that represents the residual energy input variable Low, medium and high are the linguistic variables of this fuzzy set. Low and high linguistic variables have a trapezoidal membership function while medium has a triangular membership function.

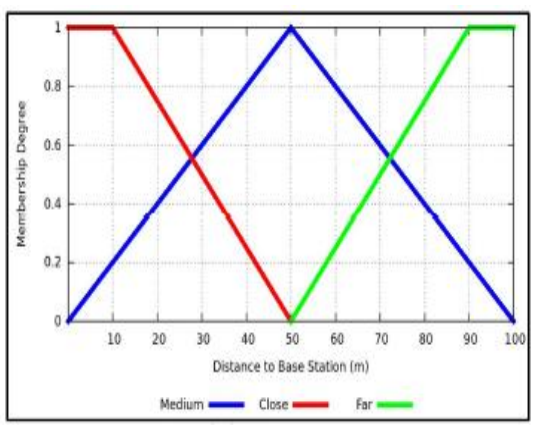

Fig. (3): Fuzzy set for fuzzy input variable Distance to Base

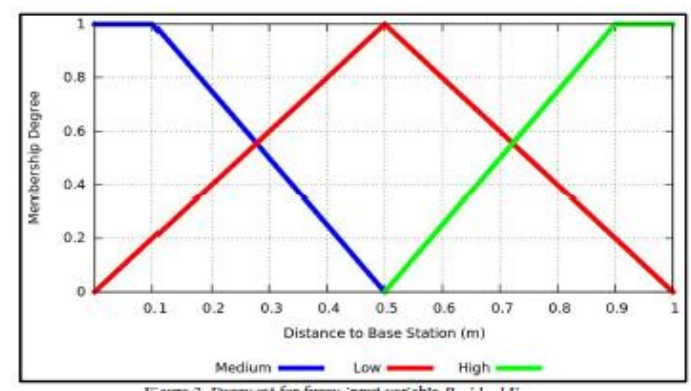

Fig. (4): Fuzzy set for fuzzy input variable Residual Energy

The output of the proposed fuzzy model represents the cluster head selection chances that represented by the fitness and it is divided into nine levels: very small, small, medium small, medium, medium large, large and very large as shown in Fig.(5). While, table (1) shows the fuzzy rule base that consists of 9 rules.

In the defuzzification module, the output of the fuzzy inference engine must be transformed to the crisp values to be used by the users.

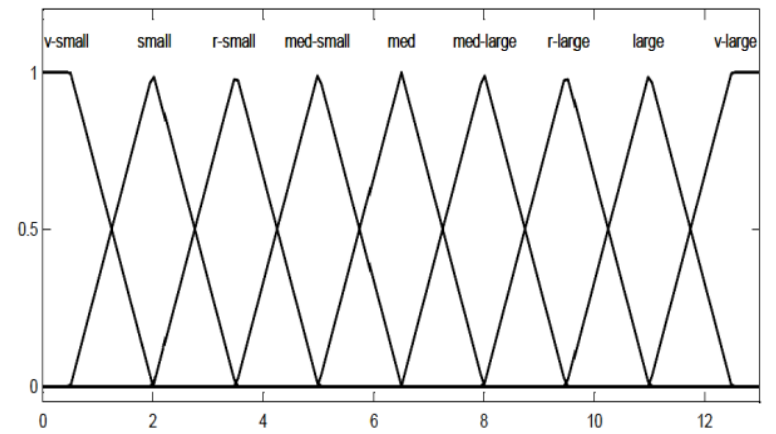

Fig.(5): Membership Functions of Cluster Head Fitness
Table (1): Fuzzy Rules used in the fuzzy proposed system

\begin{tabular}{|c|c|c|c|}
\hline $\begin{array}{c}\text { Rule } \\
\text { No. }\end{array}$ & $\begin{array}{c}\text { Distance to } \\
\text { Base }\end{array}$ & $\begin{array}{c}\text { Residual } \\
\text { Energy }\end{array}$ & The output \\
\hline 1 & Close & Low & Very Small \\
\hline 2 & Close & Medium & Small \\
\hline 3 & Close & Medium & Small \\
\hline 4 & Medium & Low & Small \\
\hline 5 & Medium & Medium & Medium \\
\hline 6 & Medium & Medium & Large \\
\hline 7 & Far & Low & Large \\
\hline 8 & Far & Medium & Large \\
\hline 9 & Far & High & Very Large \\
\hline
\end{tabular}

However, the present research can increase the lifetime and optimize the energy management of the WSNs.

\section{SIMULATION OF THE RESULTS}

The proposed fuzzy system is applied for a simulated WSN. The simulation processes are carried out in MATLAB. 50 sensor nodes are randomly deployed in a topographical area of dimension $100 \mathrm{~m} \times 100 \mathrm{~m}$. All nodes have the same initial energy 2J. Simulated WSN is used in communicating and sensing the environment parameters of the radiation system, and transmitted them for its control centre. This radiation system is used for radiating the products with pre-determined doses based on their properties to improve their properties. This network contains many sensors that must be used to determine the level of the water around the radiation source, the temperature, the pressure inside the radiation area, the closing of the door, etc. The reliable operation of this important and critical radiated process is depended mainly on the accuracy and speeding and the lifetime of transmitting the sensors' data. Therefore, using the proposed system can improve the important parameters rather than the traditional LEACH clustering algorithm used for the WSNs.

To evaluate the performance of the proposed system, a simulation for the applied WSN case of study is run. Then, its results are compared with those obtained from the traditional common LEACH algorithm for WSNs.

Figure (6-8) represents the results of the comparisons between the transmitting time for the data inside the WSNs, lifetime, and the accuracy of the two algorithms under the same conditions. 


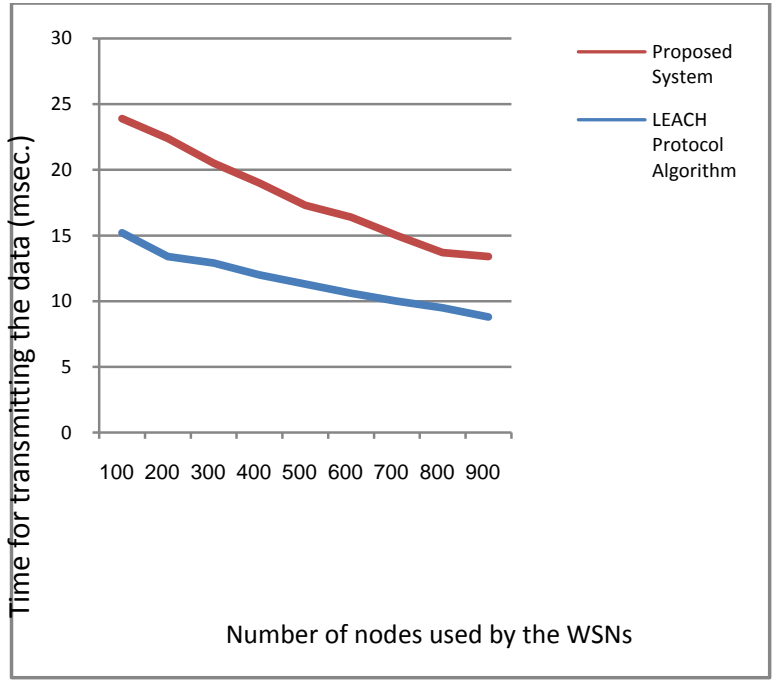

Fig. (6): A comparison between the transmutation time for LEACH and the proposed system for the same WSN.

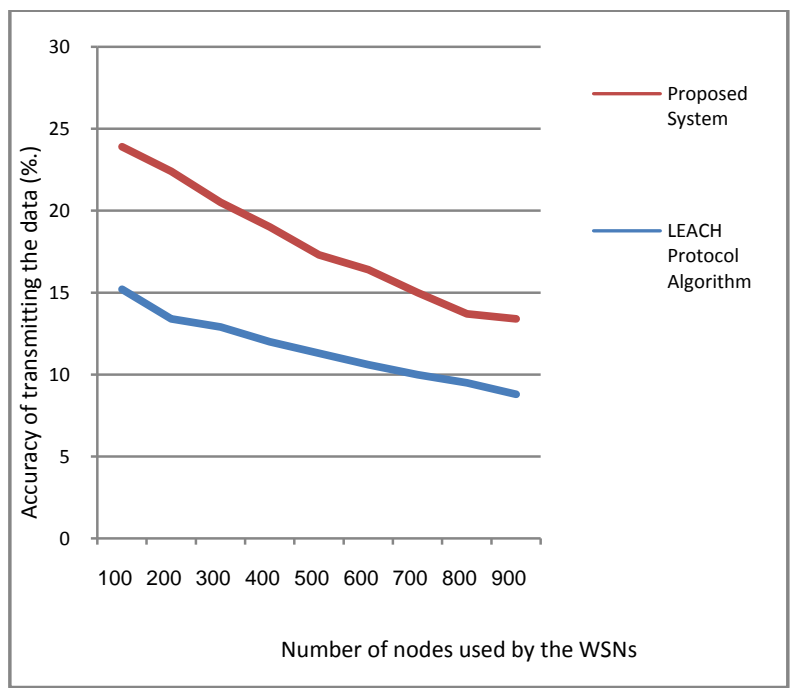

Fig. (7): A comparison between the accuracy for LEACH and the proposed system for the same WSN.

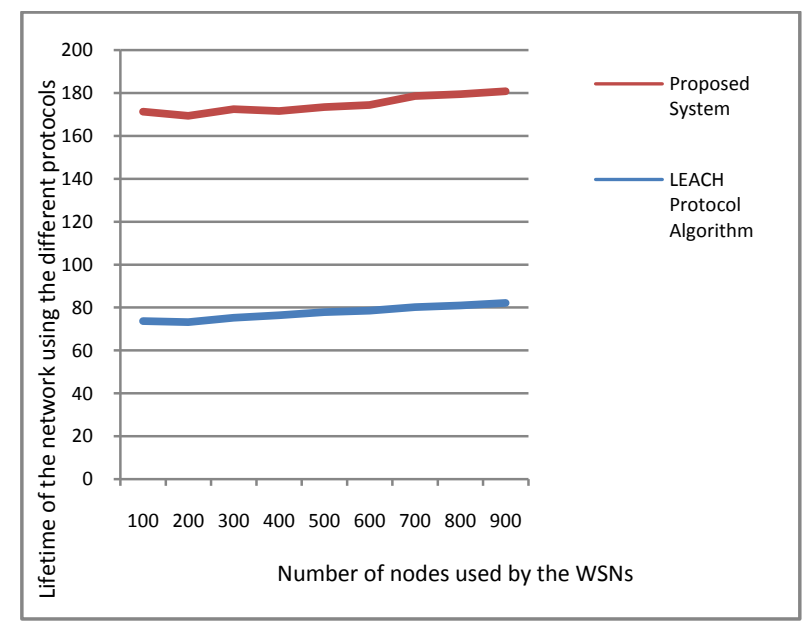

Fig. (8): A comparison between the lifetime for LEACH and the proposed system for the same WSN.

From the obtained results, there is a significant improving of the lifetime, accuracy and speeding of the transmission time of the WSN by incorporating the fuzzy modeling into the common LEACH Protocol.

\section{CONCLUSION}

In the recent years, there are great uses of the wireless sensor networks in many industrial and engineering applications.

LEACH protocol proved its accepted performance when applied by various types of WSNs. But, it still suffers from some limitations.

The proposed system has introduced the uses of fuzzy logic modeling incorporated inside the operation of LEACH Protocol. The proposed fuzzy system of the WSNs has the following advantages:

(1) handle the uncertainties data in their operations,

(2) integrate many different parameters to select the clusterheads,

(3) can select the cluster-head that has the greatest chance in its near area.

(4) select the most suitable heads among the other sensor nodes.

(5) do fast adaptation of the network' values.

(6) have an energy balancing between the nodes

(7) select the minimum power consumption of the routing paths .

(8) speed up the time for the WSN' Operation.

The suggested system is evaluated by comparing its obtained results with those having from applying the LEACH protocol under the same conditions.

It is found that, the proposed system has proved its goodness for applicability for the real-time applications.

\section{REFERENCES}

[1] Akyildiz F., (2002), "A Survey on Sensor Networks," Computer Journal of IEEE Communications, Magazine, 40 (8):102-114

[2] Zhang, Y.; Li, W. (2012); Modeling and energy consumption evaluation of a stochastic wireless sensor network, Eurasip J Wirel Comm, ISSN 1687-1499, 2012(1): 1-11.

[3] Hadjila, M., Guyennet, H., Feham, M., (2013), EnergyEfficient in wireless sensor networks using fuzzy CMeans clustering approach, International Journal of Sensors and Sensor Networks, 1(2) : 21-26

[4] Huruiala, P.-C. Urzica, A. and Gheorghe, L. , (2010), "Hierarchical routing protocol based on evolutionary algorithms for Wireless Sensor Networks," in Proc. 9th Roedunet Int. Conf. (RoEduNet), 2010, pp. 387-392.

[5] Taruna, S., Kusum Jain and Purohit, G.N., (2011), "Power Efficient Clustering Protocol (PECP)Heterogeneous Wireless Sensor Network," International Journal of Wireless \& Mobile Networks (IJWMN) vol.3, no.3, June 2011.

[6] Zhang, Y. Q. and Wei, L. , (2010) "Improving the LEACH protocol for wireless sensor networks," in Proc. IET-WSN Wireless Sensor Network IET Int. Conf, 2010, pp. 355-359. 
[7] Godbole, V., (2012), Performance Analysis of Clustering Protocol Using Fuzzy Logic for Wireless Sensor Network, IAES International Journal of Artificial Intelligence (IJ-AI), 1(3): 103-111.

[8] H. Bagci and A. Yazici, "An energy aware fuzzy unequal clustering algorithm for wireless sensor networks," in Proc. IEEE Int Fuzzy Systems (FUZZ) Conf, 2010, pp. 1-8.

[9] Akyildiz F., (2002), "A Survey on Sensor Networks," Computer Journal of IEEE Communications, Magazine, 40 (8):102-114.

[10] Heinzelman, A. Chandrakasan and H. Balakrishnan, An Application-Specific Protocol Architecture For Wireless Microsensor Networks, in IEEE Transactions on Wireless communications, pp. 660 -670, Oct 2002.

[11] Lee, J.-S. and Cheng, W.-L. , (2012) , "Fuzzy-LogicBased Clustering Approach for Wireless Sensor Networks Using Energy Predication," IEEE Sensors Journal, 12 (9):2891-2897.

[12] Al-Jarrah, R., A. Al-Jarrah, M., Roth, H., (2016), WSNs and on-Board Visual Fuzzy Servoing on Blimp Robot for Tracking Purposes, International Journal of Computer and Communication Engineering, 5(3):215221.
[13] Samarasooriya V. N. S. and Varshney, P. K. (2000), "A fuzzy modeling approach to decision fusion under uncertainty," Fuzzy Sets Syst., vol. 114,pp. 59-69.

[14] Gharajeh, M.S. , (2014), Determining the State of the Sensor Nodes Based on Fuzzy Theory in WSNs, International Journal of Computers Communications \& Control, 9(4):419-429.

[15] Manjunatha, P., Verma, A. and Srividya, A., (2008), "Multi-sensor data fusion in cluster based wireless sensor networks using fuzzy logic method," in Industrial and Information Systems, 2008.ICIIS2008.IEEE Region 10 and the Third international Conference on, 2008, pp. $1-6$.

[16] Chang H., Leandros S., and Tassiulas L., "Maximum Lifetime Routing in Wireless Sensor Networks," Computer Journal of IEEE/ACM Transactions on Networking, vol. 12, no. 4, pp. 609-619, 2004.

[17] Gu, S. , Yue, Y., Maple, C. and Wu, C. , (2012), "Fuzzy logic based localization in Wireless Sensor Networks for disaster environments," Proc. Int. Conf. on Automation and Computing, pp. 1-5.

[18] http://www.dma.fi.upm.es 